\title{
Efficacy of dual progesterone administration (intramuscular and vaginal) for luteal support in fresh day 3 or day 4 embryo transfer cycles
}

\author{
Eun Jee Nho ${ }^{1, *}$, Yeon Hee Hong ${ }^{1,2, *}$, Ju Hee Park ${ }^{2,3}$, Seul Ki Kim ${ }^{1,2}$, Jung Ryeol Lee ${ }^{1,2}$, Byung Chul Jee ${ }^{1,2}$, Seok Hyun Kim ${ }^{2}$ \\ 'Department of Obstetrics and Gynecology, Seoul National University Bundang Hospital, Seongnam; ${ }^{2}$ Department of Obstetrics and Gynecology, \\ Seoul National University College of Medicine, Seoul; ${ }^{3}$ Sarang I Infertility Clinic, Seoul, Korea
}

\begin{abstract}
Objective: The aim of this study was to compare in vitro fertilization outcomes between fresh day 3 or day 4 embryo transfer cycles with dual progesterone (P) administration (intramuscular and vaginal) and cycles with single intramuscular $\mathrm{P}$ administration for luteal support.

Methods: We selected 124 cycles from 100 women (under age 40 years) who underwent oocyte pick-up (number of trials $\leq 3,4-14$ oocytes obtained) and transfer of two or three day 3 or day 4 embryos at two infertility centers from January 2014 to June 2019. Dual P (intramuscular P [50 mg] daily+vaginal P) was used in 52 cycles and a single intramuscular administration of P (50 mg daily) was used in 72 cycles.

Results: Women's age, infertility factors, number of oocytes retrieved, number of transferred embryos, and mean embryo score were similar between the dual P group and the single P group. Although the number of trial cycles was significantly higher (1.9vs. 1.5), and the mean endometrial thickness on the trigger day $(10.0 \mathrm{~mm}$ vs. $11.0 \mathrm{~mm})$ was significantly lower in the dual P group, the implantation rate, clinical pregnancy rate, ongoing pregnancy rate, and miscarriage rate for both day 3 and day 4 transfers were similar between the two groups.

Conclusion: In fresh day 3 or day 4 embryo transfer cycles, dual P administration did not demonstrate any clinical advantages. Intramuscular $P$ alone appears to be sufficient for luteal support.
\end{abstract}

Keywords: Embryo transfer; Pregnancy; Progesterone

\section{Introduction}

In fresh embryo transfer (ET) cycles, luteal support is a routine procedure because it is known to enhance the clinical pregnancy rate, ongoing pregnancy rate, and live birth rate [1]. The main rationale is that pituitary suppression during ovarian stimulation negatively affects corpus luteum function, leading to decreased progesterone (P)

Received: December 18, 2019 Revised: February 7, 2020 · Accepted: March 16, 2020 Corresponding author: Byung Chul Jee

Department of Obstetrics and Gynecology, Seoul National University Bundang Hospital, 82 Gumi-ro 173beon-gil, Bundang-gu, Seongnam 13620, Korea Tel: +82-31-787-7254 Fax: +82-31-787-4054 E-mail: blasto@snubh.org

*These authors contributed equally to this article.

This is an Open Access article distributed under the terms of the Creative Commons Attribution Non-Commercial License (http://creativecommons.org/licenses/by-nc/4.0/) which permits unrestricted non-commercial use, distribution, and reproduction in any medium, provided the original work is properly cited. levels. Altered P levels could compromise embryo implantation potential. The oocyte pick-up procedure results in the loss of some granulosa cells, which may lead to impaired corpus luteum function [2].

Administration of $P$, which is now the main method of luteal support, can be done by intramuscular, subcutaneous, vaginal, and oral routes. The intramuscular route generally results in a higher serum $P$ level than the vaginal route, but serum $P$ levels may not reflect actual $P$ levels in the endometrial tissue [3-5]. Intramuscular P administration can frequently cause pain and, in rare cases, abscess formation. In contrast, the vaginal route avoids the first-pass metabolism of $\mathrm{P}$ in the liver, resulting in high actual P levels in the endometrial tissue [3]. Both intramuscular and vaginal administration of $P$ has been reported to yield similar clinical pregnancy rates and miscarriage rates in in vitro fertilization (IVF) cycles [1,6]. Various vaginal formulations (gels, 
inserts, rings, and suppositories) have also been reported to lead to similar clinical pregnancy rates and live birth rates $[7,8]$.

Recently, attempts have been made to combine several forms of $P$ to provide more intense luteal support. The combination of intramuscular and vaginal $P$ is used by some clinicians, but little is known about its efficacy [4]. In a study by Pabuccu et al. [4], women who received dual luteal support (intramuscular P [50 mg]+vaginal P [200 $\mathrm{mg}$ ] three times) had a significantly higher implantation rate than those who received vaginal $P$ only, but the clinical pregnancy rate and ongoing pregnancy rate were similar [4]. No study has yet compared fresh ET cycle outcomes between patients who receive intramuscular $P$ and vaginal $P$ and those who receive intramuscular $P$ alone.

At our centers, some clinicians prefer a combination of intramuscu$\operatorname{lar} P$ (50 mg daily) and vaginal $P$ with various formulas as luteal support in fresh ET cycles. Thus we retrospectively compared the clinical efficacy of fresh day 3 or day 4 ET cycles with dual P administration (intramuscular and vaginal) and cycles with intramuscular $\mathrm{P}$ alone as luteal support.

\section{Methods}

We selected 124 cycles from 100 women who underwent oocyte pick-up at two infertility centers (84 cycles at Seoul National University Bundang Hospital and 40 cycles at Sarang I Infertility Clinic) from January 2014 to June 2019. The inclusion criteria were (1) women under age 40 years; (2) first, second, or third IVF-ET trials; (3) 4-14 oocytes obtained; and (4) two or three day 3 or day 4 embryos transferred. The Institutional Review Board of the Seoul National Bundang Hospital approved this study (IRB No. B-1912-582-111).

Ovarian stimulation was performed using recombinant folliclestimulating hormone (FSH; Gonal-F; Merck-Serono, Darmstadt, Germany), recombinant FSH with recombinant luteinizing hormone (Pergoveris, Merck-Serono), or purified human menopausal gonadotropin (Menopur, Ferring, Germany). Pituitary suppression was performed by a flexible gonadotropin-releasing hormone $(\mathrm{GnRH})$ antagonist protocol (118 cycles) or a luteal long protocol of GnRH agonist (6 cycles). Once ultrasound monitoring revealed two or more follicles $\geq 18 \mathrm{~mm}$ in diameter, $250 \mu \mathrm{g}$ of recombinant human chorionic gonadotrophin (hCG; Ovidrel, Merck-Serono) was given. Oocyte pickup was performed 36-38 hours after the hCG injection.

Starting on the day of oocyte pick-up, luteal support was started. Dual P (intramuscular and vaginal) was used in 52 cycles ( 12 cycles at Seoul National University Bundang Hospital and 40 cycles at Sarang I Infertility Clinic) and single intramuscular $P$ alone was used in 72 cycles. In the dual P group, 50 mg of intramuscular P (Sugest, Sanzyme, Hyderabad, India) daily and 100 mg of vaginal utrogestan (Lutinus,
Ferring) two times every other day were used. In the single intramuscular P group, a daily dose of $50 \mathrm{mg}$ of Sugest or Genifer P (Watson Laboratories Inc., Parsippany, NJ, USA) or Taiyu P (Jaytech Biogen, Seoul, Korea) was used. Luteal support was continued until 8-9 gestational weeks, if pregnant.

Mature oocytes were inseminated by the conventional method (60 cycles), intracytoplasmic sperm injection (48 cycles), or combined methods (16 cycles). Fertilization was confirmed the next day by observing two pronuclei. Embryo quality was evaluated by morphological criteria based on the degree of fragmentation and the regularity of blastomeres on day 3 or day 4 after insemination. Embryos were graded as follows: grade $A, 0 \%$ anucleate fragments, regularity of blastomeres, no apparent morphological abnormality; grade $B$, $<20 \%$ anucleate fragments, regularity of blastomeres, no apparent morphological abnormality; grade C, 20\%-50\% anucleate fragments, irregularity of blastomeres, no apparent morphological abnormality; and grade D, $>50 \%$ anucleate fragments, irregularity of blastomeres, and apparent morphological abnormalities.

Two or three embryos were transferred on day 3 (109 cycles) or day 4 (15 cycles). At the time of ET, the embryo was scored using the Steer method [9]. After multiplying the cell number by the grading score $(A, 4 ; B, 3 ; C, 2 ; D, 1)$, each score was summed. The mean embryo score was obtained as the summed embryo score divided by the number of embryos. A compaction was considered as a superstaged 8-cell with a score of 5 and a morula was considered as a 12 cell with a score of 6 .

Serum hCG levels were determined 14 days after oocyte pick-up. Clinical pregnancy was defined as visibility of an intrauterine gestational sac. Ongoing pregnancy was defined as clinical pregnancy persisting beyond 12 gestational weeks. Miscarriage was defined as termination of clinical pregnancy before 12 gestational weeks. The implantation rate was calculated as the number of gestational sacs divided by the number of embryos transferred.

Statistical analysis was performed using SPSS ver. 25 (IBM Corp., Armonk, NY, USA). The Student $t$-test or the Mann-Whitney U-test was used as appropriate to compare numerical data between the two groups. The chi-square test or the Fisher exact test was used to compare ratios. Multiple logistic analysis was performed to identify specific parameters that affected clinical pregnancy. Receiver operating characteristic curve analysis was performed to obtain a cutoff value for a specific parameter. A $p$-value $<0.05$ was considered to indicate statistical significance.

\section{Results}

Patients' demographic characteristics and cycle outcomes are presented in Table 1. Women's age, infertility factors, the number of oo- 
Table 1. Demographic and cycle information in the dual progesterone support group and the single progesterone support group

\begin{tabular}{|c|c|c|c|}
\hline Variable & $\begin{array}{l}\text { Dual progesterone support group } \\
\text { (52 cycles) }\end{array}$ & $\begin{array}{l}\text { Single progesterone support group } \\
\text { (72 cycles) }\end{array}$ & $p$-value ${ }^{a}$ \\
\hline Women's age (yr) & $35.5 \pm 2.9$ & $34.4 \pm 3.4$ & 0.071 \\
\hline Body mass index $\left(\mathrm{kg} / \mathrm{m}^{2}\right)$ & $22.8 \pm 3.7$ & $22.5 \pm 4.0$ & 0.671 \\
\hline $\mathrm{AMH}(\mathrm{ng} / \mathrm{mL})$ & $3.2 \pm 2.2$ & $3.5 \pm 2.4$ & 0.476 \\
\hline Indication & & & 0.355 \\
\hline Female factor & $15(28.8)$ & $28(38.9)$ & \\
\hline Male factor & $16(30.8)$ & $15(20.8)$ & \\
\hline Other & $21(40.4)$ & $29(40.3)$ & \\
\hline No. of trial cycles & $1.9 \pm 0.8$ & $1.5 \pm 0.6$ & 0.005 \\
\hline No. of oocytes & $7.8 \pm 3.6$ & $7.8 \pm 2.8$ & 0.971 \\
\hline Maximal endometrial thickness (mm) & $10.0 \pm 1.8$ & $11.0 \pm 2.9$ & 0.030 \\
\hline Embryo transfer day & & & 0.201 \\
\hline Day 3 & $48(92.3)$ & $61(84.7)$ & \\
\hline Day 4 & $4(7.7)$ & $11(15.3)$ & \\
\hline \multicolumn{4}{|l|}{ No. of embryos transferred } \\
\hline Day 3 transfer & $2.5 \pm 0.5$ & $2.3 \pm 0.5$ & 0.056 \\
\hline Day 4 transfer & $2.5 \pm 0.6$ & $2.4 \pm 0.5$ & 0.662 \\
\hline \multicolumn{4}{|l|}{ Mean embryo score } \\
\hline Day 3 transfer & $24.7 \pm 9.6$ & $27.2 \pm 7.7$ & 0.138 \\
\hline Day 4 transfer & $40.8 \pm 11.1$ & $41.6 \pm 24.0$ & 0.955 \\
\hline \multicolumn{4}{|l|}{ Implantation rate } \\
\hline Day 3 transfer & $12.7(15 / 118)$ & $15.8(22 / 139)$ & 0.489 \\
\hline Day 4 transfer & $10(1 / 10)$ & $15.4(4 / 26)$ & 0.407 \\
\hline \multicolumn{4}{|l|}{ Clinical pregnancy rate } \\
\hline Day 3 transfer & $25(12 / 48)$ & $31.1(19 / 61)$ & 0.480 \\
\hline Day 4 transfer & $25(1 / 4)$ & $36.4(4 / 11)$ & 1.000 \\
\hline \multicolumn{4}{|l|}{ Ongoing pregnancy rate } \\
\hline Day 3 transfer & $18.8(9 / 48)$ & $27.9(17 / 61)$ & 0.267 \\
\hline Day 4 transfer & $25(1 / 4)$ & $36.4(4 / 11)$ & 1.00 \\
\hline \multicolumn{4}{|l|}{ Miscarriage rate } \\
\hline Day 3 transfer & $25(3 / 12)$ & $10.5(2 / 19)$ & 0.350 \\
\hline Day 4 transfer & $0(0 / 1)$ & $0(0 / 4)$ & - \\
\hline
\end{tabular}

Values are presented as mean \pm standard deviation, number (\%), or percent (number).

$\mathrm{AMH}$, anti-Müllerian hormone.

a)Student $t$-test.

cytes retrieved, and the number of embryos transferred were similar between the dual $\mathrm{P}$ group and the single $\mathrm{P}$ group. However, the number of trial cycles was significantly higher, and the mean endometrial thickness on the trigger day was significantly lower, in the dual $P$ group. Nonetheless, the implantation rate, clinical pregnancy rate, ongoing pregnancy rate, and miscarriage rate, both for day 3 and day 4 transfers, were similar between the two groups.

Multiple logistic analysis revealed that maximal endometrial thickness had a significant effect on clinical pregnancy (Table 2). Women's age, trial cycle number, ET day, the number of embryos transferred, the method of luteal support, and the infertility center were not significant factors. The best cut-off value of maximal endometrial thickness for predicting clinical pregnancy was $10.65 \mathrm{~mm}$ (area under the curve, $0.643 ; 95 \%$ confidence interval, $0.537-0.749 ; p=0.013$; sensi-
Table 2. Multiple logistic analysis of factors affecting clinical pregnancy

\begin{tabular}{lcc}
\hline Parameter & $\begin{array}{c}\text { Linear correlation } \\
\text { coefficient }(r)\end{array}$ & $p$-value \\
\hline Women's age & 0.009 & 0.551 \\
Trial cycle number & 0.070 & 0.290 \\
Maximal endometrial thickness & 0.046 & 0.035 \\
Embryo transfer day & -0.007 & 0.956 \\
No. of embryos transferred & -0.150 & 0.154 \\
Method of luteal support & -0.114 & 0.459 \\
Infertility center & -0.200 & 0.201 \\
\hline
\end{tabular}

tivity, 63.9\%; specificity, 63.2\%).

A subgroup analysis was performed according to the maximal endometrial thickness cutoff of $10.65 \mathrm{~mm}$ (Table 3). In cycles with a 
Table 3. Subgroup analysis according to maximal endometrial thickness

\begin{tabular}{|c|c|c|c|c|c|c|}
\hline \multirow[b]{2}{*}{ Variable } & \multicolumn{3}{|c|}{ Maximal endometrial thickness $<10.65 \mathrm{~mm}$} & \multicolumn{3}{|c|}{ Maximal endometrial thickness $\geq 10.65 \mathrm{~mm}$} \\
\hline & $\begin{array}{l}\text { Dual progesterone } \\
\text { group (34 cycles) }\end{array}$ & $\begin{array}{l}\text { Single progesterone } \\
\text { group (34 cycles) }\end{array}$ & $p$-value & $\begin{array}{l}\text { Dual progesterone } \\
\text { group (18 cycles) }\end{array}$ & $\begin{array}{l}\text { Single progesterone } \\
\text { group (38 cycles) }\end{array}$ & $p$-value ${ }^{a)}$ \\
\hline Women's age (yr) & $36(33.8-37)$ & $34.5(32-38)$ & 0.430 & $36.5(33.8-39)$ & $35(32-36)$ & 0.095 \\
\hline Body mass index $\left(\mathrm{kg} / \mathrm{m}^{2}\right)$ & $22.5(20.6-25.9)$ & $21.3(20.3-24.2)$ & 0.094 & $21.3(18.7-23.7)$ & $22.4(19.5-25.6)$ & 0.319 \\
\hline $\mathrm{AMH}(\mathrm{ng} / \mathrm{mL})$ & $2.6(1.9-4)$ & $2.5(1.3-4.4)$ & 0.650 & $1.9(1.6-4.7)$ & $3.6(2.3-4.2)$ & 0.181 \\
\hline Indication & & & 0.788 & & & 0.336 \\
\hline Female factor & $11(32.4)$ & $12(35.3)$ & & $4(22.2)$ & $16(42.1)$ & \\
\hline Male factor & $9(26.5)$ & $5(14.7)$ & & $7(38.9)$ & $10(26.3)$ & \\
\hline Other & $14(41.2)$ & $17(50)$ & & $7(38.9)$ & $12(31.6)$ & \\
\hline No. of trial cycles & $2(1-3)$ & $1(1-2)$ & 0.029 & $1.5(1-3)$ & $1(1-2)$ & 0.207 \\
\hline No. of oocytes & $6.5(5-10.5)$ & $7(5-10.5)$ & 0.635 & $7(5.8-11)$ & $8(6-10)$ & 0.923 \\
\hline Maximal endometrial thickness $(\mathrm{mm})$ & $9(8.2-9.9)$ & $8.9(7.8-10.1)$ & 0.435 & $11.2(11.2-13.1)$ & $12.1(11.3-13.3)$ & 0.307 \\
\hline \multicolumn{7}{|l|}{ Embryo transfer day } \\
\hline Day 3 & $32(94.1)$ & $30(88.2)$ & & $16(88.9)$ & 31 (81.6) & \\
\hline Day 4 & $2(5.9)$ & $4(11.8)$ & & $2(11.1)$ & $7(18.4)$ & \\
\hline \multicolumn{7}{|l|}{ No. of embryos transferred } \\
\hline Day 3 transfer & $2(2-3)$ & $2(2-2.3)$ & 0.149 & $3(2-3)$ & $2(2-3)$ & 0.116 \\
\hline Day 4 transfer & 2.5 & $2.5(2-3)$ & 1.000 & 2.5 & $2(2-3)$ & 0.667 \\
\hline \multicolumn{7}{|l|}{ Mean embryo score } \\
\hline Day 3 transfer & $24(20-26)$ & $26.8(19.8-32)$ & 0.083 & $24(18-31.3)$ & $28(24-32)$ & 0.167 \\
\hline Day 4 transfer & 24.9 & $30.3(16.5-44.7)$ & 0.800 & 34 & $39(22-48)$ & 0.889 \\
\hline \multicolumn{7}{|l|}{ Implantation rate } \\
\hline Day 3 transfer & $6.4(5 / 77)$ & $27(10 / 37)$ & 0.146 & $24.4(8 / 41)$ & $16.7(12 / 72)$ & 0.353 \\
\hline Day 4 transfer & $0(0 / 5)$ & $10(1 / 10)$ & 0.800 & $20(1 / 5)$ & $18.8(3 / 16)$ & 0.889 \\
\hline \multicolumn{7}{|l|}{ Clinical pregnancy rate } \\
\hline Day 3 transfer & $12.5(4 / 32)$ & $26.7(8 / 30)$ & 0.158 & $50(8 / 16)$ & $35.5(11 / 31)$ & 0.337 \\
\hline Day 4 transfer & $0(0 / 2)$ & $25(1 / 4)$ & 1.000 & $50(1 / 2)$ & $42.9(3 / 7)$ & 1.000 \\
\hline \multicolumn{7}{|l|}{ Ongoing pregnancy rate } \\
\hline Day 3 transfer & $9.4(3 / 32)$ & $23.3(7 / 30)$ & 0.176 & $37.5(6 / 16)$ & $32.3(10 / 31)$ & 0.719 \\
\hline Day 4 transfer & $0(0 / 2)$ & $25(1 / 4)$ & 1.000 & $50(1 / 2)$ & $42.9(3 / 7)$ & 1.000 \\
\hline \multicolumn{7}{|l|}{ Miscarriage rate } \\
\hline Day 3 transfer & $25(1 / 4)$ & $12.5(1 / 8)$ & 1.000 & $25(2 / 8)$ & $9.1(1 / 11)$ & 0.546 \\
\hline Day 4 transfer & - & $0(0 / 1)$ & - & $0(0 / 1)$ & $0(0 / 3)$ & - \\
\hline
\end{tabular}

Values are presented as median (interquartile range) or number (\%).

AMH, anti-Müllerian hormone.

a) Mann-Whitney U-test.

maximal endometrial thickness $<10.65 \mathrm{~mm}$ and $\geq 10.65 \mathrm{~mm}$, the implantation rate, clinical pregnancy rate, ongoing pregnancy rate, and miscarriage rate were also similar between the dual and single $P$ groups.

\section{Discussion}

In the present study, the IVF outcomes were similar between fresh day 3 or day $4 \mathrm{ET}$ cycles with dual P support and cycles with single intramuscular $P$ support. Therefore, intramuscular $P$ alone appears to be sufficient for luteal support. This indicates that intramuscular $P$ alone is sufficient to achieve physiologically necessary P levels.

Intramuscular P generally results in higher serum $P$ levels than vagi- nal P [3-5]. In fresh IVF-ET cycles, the serum P level during the mid-luteal phase has been reported to be $94.3 \pm 8.8 \mathrm{ng} / \mathrm{mL}$ when intramuscular (P $100 \mathrm{mg}$ ) was administered and $57.7 \pm 7.4 \mathrm{ng} / \mathrm{mL}$ when a vaginal $8 \%$ progesterone gel was used $(p<0.01)$ [10]. Although the actual $\mathrm{P}$ level in the endometrial tissue may be higher after vaginal $\mathrm{P}$ than after intramuscular $\mathrm{P}$, our findings suggest that $\mathrm{P}$ might work well in the endometrial tissue after intramuscular $\mathrm{P}$ administration, similar to after vaginal $P$ administration.

In non-stimulated frozen $E T$ cycles, the absolute effects of $P$ administration can be identified, because the corpus luteum is absent [11]. In our experience, the serum P level at the time of frozen ET was 35 $\mathrm{ng} / \mathrm{mL}$ after intramuscular $\mathrm{P}$ (Sugest; $50 \mathrm{mg} /$ day) was administered for 3 days. A recent study reported that women with serum $P$ levels 
$<10 \mathrm{ng} / \mathrm{mL}$ at the time of frozen ET showed a significantly lower clinical pregnancy rate and live birth rate than those with levels $\geq 10$ $\mathrm{ng} / \mathrm{mL}$ [12]. Another study indicated that women with serum P levels $<10.64 \mathrm{ng} / \mathrm{mL}$ before the day of frozen ET showed a significantly lower live birth rate and significantly higher miscarriage rate [13]. In donor cycles, recipients with serum P levels $<20 \mathrm{ng} / \mathrm{mL}$ at the time of ET were less likely to achieve clinical pregnancy [14].

In fresh ET cycles, it is difficult to assess the absolute physiological $P$ level due to the growth of the corpus luteum and other iatrogenic factors, such as pituitary suppression, controlled ovarian stimulation, and the ovum pick-up procedure [2]. Therefore, further research is needed to determine the lowest effective serum $P$ level in fresh ET cycles. Intramuscular $P$ alone appears to be sufficient to lower uterine contractility and endometrial wave activity. Uterine contractility was reported to significantly decrease after intramuscular $P$ administration in frozen ET cycles [11]. Furthermore, intramuscular $P$ has a longer half-life (over 1 day) than vaginal $P$; therefore, it can effectively maintain uterine quiescence [11].

As far as we know, this is the first study to compare the clinical efficacy of cycles with dual $\mathrm{P}$ administration (intramuscular and vaginal) and cycles with intramuscular $P$ alone as luteal support in fresh ET cycles. We included only fresh cycles with two or three embryos transferred on day 3 or day 4 to avoid the possible confounding effect induced by multiple ET.

The main limitation of this study is that intramuscular $P$ alone was used at only one center. Differences in the culture conditions and personnel at the two centers may have affected the pregnancy rate. A further randomized trial could confirm our findings. In conclusion, fresh IVF outcomes were similar between fresh day 3 or day 4 ET cycles with dual $P$ support and cycles with single intramuscular $P$ support. Therefore, intramuscular $\mathrm{P}$ alone appears to be sufficient for luteal support.

\section{Conflict of interest}

Byung Chul Jee has been an editor of Journal of Clinical and Experimental Reproductive Medicine since 2018; however, he was not involved in the peer reviewer selection, evaluation, or decision process of this article. No other potential conflict of interest relevant to this article was reported.

\section{ORCID}

$\begin{array}{ll}\text { Eun Jee Nho } & \text { https://orcid.org/0000-0002-8214-1434 } \\ \text { Yeon Hee Hong } & \text { https://orcid.org/0000-0002-9709-4175 } \\ \text { Ju Hee Park } & \text { https://orcid.org/0000-0001-5094-1640 } \\ \text { Seul Ki Kim } & \text { https://orcid.org/0000-0002-1647-6711 }\end{array}$

Jung Ryeol Lee https://orcid.org/0000-0003-3743-2934

Byung Chul Jee https://orcid.org/0000-0003-2289-6090

Seok Hyun Kim https://orcid.org/0000-0003-0649-3224

\section{Author contributions}

Conceptualization: BCJ. Data curation: all authors. Formal analysis: BCJ, EJN, YHH, JHP. Methodology: BCJ, EJN, YHH, SKK, JRL. Project administration: $B C J, E J N, Y H H, S K K$, JRL. Writing-original draft: BCJ, EJN, YHH. Writing-review \& editing: all authors.

\section{References}

1. van der Linden M, Buckingham K, Farquhar C, Kremer JA, Metwally M. Luteal phase support for assisted reproduction cycles. Cochrane Database Syst Rev 2015;2015:CD009154.

2. Fatemi HM, Popovic-Todorovic B, Papanikolaou E, Donoso P, Devroey P. An update of luteal phase support in stimulated IVF cycles. Hum Reprod Update 2007;13:581-90.

3. Shapiro D, Boostanfar R, Silverberg K, Yanushpolsky EH. Examining the evidence: progesterone supplementation during fresh and frozen embryo transfer. Reprod Biomed Online 2014;29 Suppl 1:S1-14.

4. Pabuccu EG, Pabuccu R, Evliyaoglu Ozdegirmenci O, Bostancı Durmus $\mathrm{A}$, Keskin M. Combined progesterone $(\mathrm{IM}+\mathrm{V})$ versus vaginal progesterone for luteal support in cleavage-stage embryo transfer cycles of good prognosis patients. Gynecol Endocrinol 2016;32:366-9.

5. Hershko Klement A, Samara N, Weintraub A, Mitri F, Bentov Y, Chang $P$, et al. Intramuscular versus vaginal progesterone administration in medicated frozen embryo transfer cycles: a randomized clinical trial assessing sub-endometrial contractions. Gynecol Obstet Invest 2018;83:40-4.

6. Zarutskie PW, Phillips JA. A meta-analysis of the route of administration of luteal phase support in assisted reproductive technology: vaginal versus intramuscular progesterone. Fertil Steril 2009;92:163-9.

7. Doody KJ, Schnell VL, Foulk RA, Miller CE, Kolb BA, Blake EJ, et al. Endometrin for luteal phase support in a randomized, controlled, open-label, prospective in-vitro fertilization trial using a combination of Menopur and Bravelle for controlled ovarian hyperstimulation. Fertil Steril 2009;91:1012-7.

8. Stadtmauer L, Silverberg KM, Ginsburg ES, Weiss H, Howard B. Progesterone vaginal ring versus vaginal gel for luteal support with in vitro fertilization: a randomized comparative study. Fertil Steril 2013;99:1543-9.

9. Steer CV, Mills CL, Tan SL, Campbell S, Edwards RG. The cumula- 
tive embryo score: a predictive embryo scoring technique to select the optimal number of embryos to transfer in an in-vitro fertilization and embryo transfer programme. Hum Reprod 1992; 7:117-9.

10. Chantilis SJ, Zeitoun KM, Patel SI, Johns DA, Madziar VA, McIntire DD. Use of Crinone vaginal progesterone gel for luteal support in in vitro fertilization cycles. Fertil Steril 1999;72:823-9.

11. Casper RF. Luteal phase support for frozen embryo transfer cycles: intramuscular or vaginal progesterone? Fertil Steril 2014;101:627-8.

12. Cedrin-Durnerin I, Isnard T, Mahdjoub S, Sonigo C, Seroka A, Comtet $\mathrm{M}$, et al. Serum progesterone concentration and live birth rate in frozen-thawed embryo transfers with hormonally prepared endometrium. Reprod Biomed Online 2019;38:472-80.

13. Gaggiotti-Marre S, Martinez F, Coll L, Garcia S, Álvarez M, Parriego $M$, et al. Low serum progesterone the day prior to frozen embryo transfer of euploid embryos is associated with significant reduction in live birth rates. Gynecol Endocrinol 2019;35:439-42.

14. Brady PC, Kaser DJ, Ginsburg ES, Ashby RK, Missmer SA, Correia $\mathrm{KF}$, et al. Serum progesterone concentration on day of embryo transfer in donor oocyte cycles. J Assist Reprod Genet 2014;31: 569-75. 